\title{
Possible variations of the fine structure constant $\alpha$ and their metrological significance
}

\author{
K.A. Bronnikov and S.A. Kononogov \\ VNIIMS, 46 Ozyornaya St., Moscow 119361, Russia
}

\begin{abstract}
We briefly review the recent experimental results on possible variations of the fine structure constant $\alpha$ on the cosmological time scale and its position dependence. We outline the theoretical grounds for the assumption that $\alpha$ might be variable, mention some phenomenological models incorporating a variable $\alpha$ into the context of modern cosmology and discuss the significance of possible $\alpha$ variations for theoretical and practical metrology.
\end{abstract}

\section{Introduction}

The idea that fundamental physical constants (FPC), including the Planck constant, the speed of light, stable particle masses and the coupling constants of the basic interactions (above all, Newton's gravitational constant $G$ and the fine structure constant $\alpha$ ) may be variable, was put forward for the first time by Dirac in 1937, soon after the advent of Friedmann's expanding Universe models (1922) and their confirmation by Hubble (1929). In the subsequent decades, the possible FPC variability in the course of cosmological evolution as well as their dependence on position in space, on the magnitude of physical fields or on spatial and energy scales occupied quite a prominent place in theoretical physics and cosmology, including many works of the VNIIMS theoretical group, Refs. [1-11] and others. In addition to the above-mentioned FPC, there appeared other "candidate variables" such as, for instance, the cosmological constant and the coupling constants of the weak and strong interactions. The basic motivation for such studies has been, and remains to be, the idea of unity of physical interactions. If the conjectured unified interaction splits in different physical conditions into specific interactions known from the experiment, the FPCs that characterize these interactions should depend on the circumstances of their manifestation, above all, on the spatial and energy scales and on the fundamental field intensities (a recent detailed discussion may be found in Ref. [10]).

Thus, it is quite common to believe that unification of all physical interactions requires a number of additional (so-called internal) space-time dimensions. At present, there is no well established way of transition from multidimensional physics to the conventional fourdimensional picture. It is, however, clear that such a transition requires some procedures (such as dimensional reduction and compactification) which connect the four-dimensional effective constants with true multidimensional constants and the characteristics of extra dimensions. Thus, their size may suffer a non-trivial evolution in time and may depend on the position in external space.

As to observational data on FPC variation, there have been very few positive results till the recent years. Thus, some researchers pointed toward a possible $G$ variability according to astronomical observations, but, to our knowledge, neither of these results were confirmed afterwards (see Ref. [10] for detail). However, the very end of the 20th century was marked 
with the advent of observational evidence [12] on the variability of the electromagnetic coupling constant, the fine structure constant

$$
\begin{aligned}
& \alpha=e^{2} /(\hbar c) \quad(\text { Gaussian units), or } \\
& \alpha=e^{2} /\left(4 \pi \varepsilon_{0} \hbar c\right) \quad \text { (SI units), }
\end{aligned}
$$

where $e$ is the electron charge, $c$ is the speed of light in vacuum and $\varepsilon_{0}$ is the permittivity of free space. This and subsequent works have made the subject of FPC variability, and above all the variability of $\alpha$, highly topical. This subject is no longer regarded purely theoretical, and nowadays it rapidly increases its experimental basis.

The electromagnetic interaction, whose intensity is characterized by the constant $\alpha$, is of primary importance for the macroscopic structure of matter and in an overwhelming majority of the observed phenomena. It is for this reason that a possible variability of $\alpha$ would lead to variability of the majority of existing references of physical quantities, including such basic references as those of length, mass, time/frequency.

The constant $\alpha$, whose CODATA-recommended value is

$$
\alpha=7.297362533(27) \times 10^{-3} \approx 1 / 137.03599
$$

(the figures in parentheses mean the uncertainty in the last two meaningful digits), is one of the most precisely measured FPC: the relative uncertainty is about $3.7 \times 10^{-9}$. Moreover, its dimensionless nature makes it independent of any conventions or systems of physical units.

In what follows, we will briefly describe the basic experimental and observational data on the possible $\alpha$ variability and outline the main characteristics of theoretical models which, to one or other extent, conform to the observations. In the last section, we formulate some conclusions and discuss the significance of this research area for theoretical and practical metrology.

\section{Empirical data on $\alpha$ variability}

A great number of relevant observations and experiments are described and discussed in the comprehensive review [13] and in the shorter articles $[14,15]$. In this section, we mention only some of the data presented there, seeming to be the most convincing, and add some estimates and inferences of the last two years.

\subsection{Laboratory data}

The laboratory bounds on $\alpha$ variability are obtained by comparing the long-term behaviour of oscillators with frequencies depending on $\alpha$ in different ways. In practice, such a comparison leads to experimental bounds on certain combinations of FPCs.

To our knowledge, the first comparisons of this kind were performed in 1974-76 by two groups independently. Turneaure and Stein [16], at Stanford University, used superconducting microwave cavities with a quality factor of about $4 \times 10^{10}$ and a resonant frequency $\sim 8.6$ $\mathrm{GHz}$. The frequencies of cavity-controlled oscillators were compared over a period of 12 days with a caesium-beam atomic clock, and the relative drift was $(-0.4 \pm 3.4) \times 10^{-14} /$ day. This led to the conclusion [16] that a possible annual variation of the product

$$
g_{p} \cdot\left(m_{e} / M\right) \cdot \alpha
$$


was less than $4.1 \times 10^{-12}$, where $g_{p}$ is the proton gyromagnetic ratio, $m_{e}$ is the electron mass and $M$ is the Cs nuclear mass.

Kolosnitsyn et al. [11], at VNIIFTRI, the Russian time-frequency standard keeper, compared the behaviour of molecular clocks operating with ammonia molecular beams and a group of caesium frequency standards. The caesium standard formed a scale for measuring the frequency of the ammonia generators. The results of measurements for six years (19641969) led to a number of estimates for $\dot{\alpha} / \alpha$, all of them constraining this variation by a few units times $10^{-11}$; the best estimate was

$$
|\dot{\alpha} / \alpha| \leq 1.1 \times 10^{-11} \mathrm{yr}^{-1}
$$

Other early results on the stability of $\alpha$ and $e$ are described in Petley's book [17].

The recent estimates of possible $\alpha$ variations have diminished the uncertainties by 4 to 5 orders of magnitude as compared with [11] due to a considerably increased stability of frequency standards. Thus, according to [18], a comparison of hyperfine transition frequencies in ${ }^{87} \mathrm{Rb}$ and ${ }^{133} \mathrm{Cs}$ over a period of about 4 years has shown that

$$
d \ln \left(\nu_{\mathrm{Rb}} / \nu_{\mathrm{Cs}}\right) / d t=(0.2 \pm 7.0) \times 10^{-16} \mathrm{yr}^{-1}
$$

at $1 \sigma$. Neglecting possible changes in the the weak and strong interaction constants and thus in the nuclear magnetic moments, it leads to

$$
\dot{\alpha} / \alpha=(-0.4 \pm 16) \times 10^{-16} \mathrm{yr}^{-1} .
$$

where $\dot{\alpha}=d \alpha / d t$. Another experiment [19] compared an electric quadrupole transition in ${ }^{199} \mathrm{Hg}^{+}$to the ground-state hyperfine splitting of ${ }^{133} \mathrm{Cs}$ over 3 years, showing that

$$
\left|d \ln \left(\nu_{\mathrm{Hg}} / \nu_{\mathrm{Cs}}\right) / d t\right|<7.0 \times 10^{-15} \mathrm{yr}^{-1}
$$

and leading to

$$
|\dot{\alpha} / \alpha|<1.2 \times 10^{-15} \mathrm{yr}^{-1}
$$

under the assumption that the gyromagnetic factor $g_{\mathrm{Cs}}$ and $m_{\mathrm{e}} / m_{\mathrm{p}}$ are invariable.

Ref. [20] reported a comparison of the absolute $1 S-2 S$ transition in atomic hydrogen to the ground state of caesium, which, combined with the results of Refs. [11,19], yielded the constraint

$$
\dot{\alpha} / \alpha=(-0.9 \pm 2.9) \times 10^{-16} \mathrm{yr}^{-1} .
$$

A comparison [21] of optical transitions in ${ }^{171} \mathrm{Yb}^{+}$with a caesium standard for 2.8 years has shown that $d \ln \left(\nu_{\mathrm{Yb}} / \nu_{\mathrm{Cs}}\right) / d t=(-1.2 \pm 4.4) \times 10^{-15} \mathrm{yr}^{-1}$ which leads to

$$
\dot{\alpha} / \alpha=(-0.3 \pm 2.0) \times 10^{-15} \mathrm{yr}^{-1} .
$$

The laboratory measurements thus lead to results which, as follows from Ref. [20], may be combined to yield very hard constraints for separate basic constants including $\alpha$. The above estimates show that the allowed variations of $\alpha$ in the modern epoch are bounded to a few units of $10^{-16}$ per year. 
Further progress in the near future is expected on the basis of improved frequency standards as well as development of new methods of atomic experiments. One such method [22] is based on crossing of atomic levels in two-electron highly charged ions of different atomic numbers $Z$. It is claimed that the effect of possible variation of $\alpha$ becomes strongly (by a factor of about 1000) enhanced when studied near such crossing points. Another method [23] is to use atomic transitions between narrow lines that have an enhanced sensitivity to a possible variation of $\alpha$. The authors present a number of such transitions and claim that their method effectively suppresses the systematic sources of uncertainty that are unavoidable in conventional high-resolution spectroscopic measurements.

An improvement of at least an order of magnitude in current $\dot{\alpha}$ estimates can be achieved in space experiments planned for 2008-2009 within the PHARAO/ACES Project of the European Space Agency [24,25]. PHARAO, the "Projet d'Horloge Atomique par Refroidissement d'Atomes en Orbite", which has started in 1993, combines laser cooling techniques and a microgravity environment in a satellite orbit, allowing the development of space clocks with unprecedented performance. Its declared objectives are, among others [24], (i) to achieve time and frequency transfer with stability better than $10^{-16}$ and (ii) to perform fundamental physics tests. Its detailed description can be found in [25].

One can also mention quite a different type of $\alpha$ variability predicted by the electroweak theory which concerns strengthening of the electromagnetic coupling with increasing momentum transfer $Q^{2}$ in particle interactions at energies approaching the electroweak unification energy [26,27]. This effect has been confirmed experimentally [26] by analyzing the processes $e^{+} e^{-} \rightarrow \mu^{+} \mu^{-}$and $e^{+} e^{-} \rightarrow \mathrm{e}^{+} \mathrm{e}^{-} \mu^{+} \mu^{-}$at the TRISTAN $e^{+} e^{-}$collider at KEK (Japan). The data were accumulated at an average center-of-mass energy of $57.77 \mathrm{GeV}$, and $\alpha$ was measured to change from its known value of $\alpha^{-1} \approx 137.0$ at $Q^{2}=0$ to

$$
\alpha^{-1}=128.5 \pm 1.8 \text { (stat) } \pm 0.7 \text { (syst) } \quad \text { at } \quad Q^{2}=(57.77 \mathrm{GeV} / c)^{2} .
$$

The latter variation is physically interpreted as a result of quantum vacuum properties (see, e.g., [27] and references therein) and manifests itself at energies much higher than those relevant to atomic spectra. It has therefore nothing to do with temporal and spatial variations of $\alpha$ at astrophysical and cosmological scales.

\subsection{Geochemical data}

Very hard constraints on variations of $\alpha$ have been obtained from studies of the so-called Oklo phenomenon combined with data on the lifetimes of long-lived radioactive isotopes.

The Oklo phenomenon is a natural nuclear reactor that operated for about 200,000 years approximately two billion years ago, which, regarding cosmological observations, corresponds to redshifts around $z \sim 0.14$. The products of its operation were discovered in 1972 at Oklo uranium mine in Gabon, West Africa. The isotopic abundances of the yields bears information on the nuclear reaction rates at that time, which in turn depended on the current values of $\alpha$. One of the key quantities measured is the ratio ${ }_{62}^{149} \mathrm{Sm} /{ }_{62}^{147} \mathrm{Sm}$ of two light isotopes of samarium which are not fission products. This ratio is of the order of 0.9 in normal samarium, but is about 0.02 in Oklo ores. Such a low value is explained by depletion of ${ }_{62}^{149} \mathrm{Sm}$ by thermal neutrons to which it was exposed while the reactor was active. The 
capture cross section of a thermal neutron by ${ }^{149} \mathrm{Sm}$, i.e., the reaction

$$
{ }^{149} \mathrm{Sm}+n \rightarrow{ }^{150} \mathrm{Sm}+\gamma
$$

has a resonant energy $E_{r} \simeq 0.0973 \mathrm{eV}$, which is a consequence of a nearly cancellation between electromagnetic and strong interaction forces [28]. A detailed analysis has shown [29] that, assuming that $E_{r}$ varied only due to an $\alpha$ dependence of the electromagnetic energy, the following constraint is valid:

$$
\Delta \alpha / \alpha=(0.15 \pm 1.05) \times 10^{-7}
$$

at $2 \sigma$ level. The accuracy of the method is explained by the tiny value of the resonant energy $E_{r} \sim 0.1 \mathrm{eV}$ compared to its sensitivity to a variation of $\alpha, d E_{r} / d \ln \alpha \sim-1 \mathrm{MeV}$, so that the expected variations are smaller than $0.1 \mathrm{eV} / 1 \mathrm{MeV} \sim 10^{-7}$.

It was later noticed [30] that two ranges of variations could be compatible with the Oklo data:

$$
\Delta \alpha / \alpha=(-0.8 \pm 1.0) \times 10^{-8}, \quad \Delta \alpha / \alpha=(8.8 \pm 0.7) \times 10^{-8},
$$

but the second range is hardly compatible with the isotopic ratio of gadolinium. Note that the first range, assuming that the time variations of $\alpha$ (if any) occur uniformly, is translated to the variation rate

$$
\dot{\alpha} / \alpha=(-0.4+0.5) \times 10^{-17} \mathrm{yr}^{-1},
$$

which is apparently the tightest of the existing constraints.

Some estimates have been recently obtained under the additional assumption that the low energy neutron spectrum is well described by a Maxwell-Boltzmann distribution [3133]. Taking into account the possible variation of the strange quark mass $m_{\mathrm{s}}$ along with the assumption that all fundamental couplings vary independently led [33] to the allowed variation $\Delta \alpha / \alpha<(1-5) \times 10^{-10}$, even more stringent than above.

Another result [34], free of poorly grounded assumptions, seems more realistic:

$$
\Delta \alpha / \alpha=(4.5+1) \times 10^{-8}, \quad \dot{\alpha} / \alpha=(2.25+0.5) \times 10^{-17} \mathrm{yr}^{-1} .
$$

The most recent $\dot{\alpha}$ estimates from the Oklo phenomenon [35] have been made on the basis of modern methods of reactor physics, a detailed computer model of zone RZ2 of the Oklo reactor and full-scale calculations using two different Monte Carlo codes: the Russian code MCU REA and the worldwide known code MCNP (USA). Both codes have produced close results. It was claimed that many details of these calculations differed from the previous work (e.g., the averaged cross-section of Sm and its dependence on the shift of resonance position due to variation of fundamental constants). Still no evidence on $\alpha$ variations was found, with the resulting constraint

$$
-4 \times 10^{-17} \mathrm{yr}^{-1}<(d \alpha / d t) / \alpha<3 \times 10^{-17} \mathrm{yr}^{-1}
$$

A further improvement in the accuracy of these limits is promised.

Some conclusions have also been made by analyzing the readioactive decay of nuclides with known $\alpha$ dependence of the decay rate. The best constraint was obtained from beta 
decay of rhenium into osmium with electron emission: as noted by Peebles and Dicke [36], an extremely low value of the decay energy, about $2.5 \mathrm{keV}$, makes it very sensitive to $\alpha$ variations. Still the constraints obtained are much weaker than (8) or (9).

Laboratory studies of meteorites have led to the result [37]

$$
\Delta \alpha / \alpha=(8 \pm 16) \times 10^{-7}
$$

for the recent 4.5 billion years, which corresponds to $z \simeq 0.45$. Translated to the changing rate (again assuming its uniformity) gives approximately

$$
\dot{\alpha} / \alpha=(2 \pm 4) \times 10^{-16} / \mathrm{yr}
$$

A shortcoming of this method is its indirect nature: the Re/Os ratio is measured in iron meteorites whose age is not determined directly. Besides, the constraint (10) only concerns the mean value of $\alpha$ for 4.5 billion years rather than its instant variation rate.

\subsection{Astrophysical data}

The first and not very confident observational indication on a possible variability of $\alpha$ has appeared from studies of remote quasars. The observed quasar absorption spectra were compared with the corresponding laboratory spectra. The details of the method are described in Refs. [12,38-41] and others.

One of the recent results [39] has been obtained by analyzing the lines of SiIV in 15 systems and improves the previous estimates by a factor of three:

$$
\Delta \alpha / \alpha=(0.15 \pm 0.43) \times 10^{-5}, \quad 1.59 \leq z \leq 2.92 .
$$

This result does not confirm $\alpha$ variations.

The many-multiplet method of Webb et al. [12,40] rests on a comparison of a few lines in different samples of sources: one compares the shifts of lines which are sensitive and insensitive to $\alpha$ variations. A recent result [20] obtained from a study of 128 systems in the range $0.5<z<3$ points at lower values of $\alpha$ in the past:

$$
\Delta \alpha / \alpha=(-0.54 \pm 0.12) \times 10^{-5}, \quad 0.5 \leq z \leq 3,
$$

thus confirming the previous conclusions $[12,40]$. The authors did not find systematic errors.

There are, however, results directly opposite to these. Thus, according to [42],

$$
\Delta \alpha / \alpha=(-0.1 \pm 1.7) \times 10^{-6}, \quad z=1.15
$$

(from an analysis of absorption lines of iron of a single quasar). Similar results have been found for a system of absorption lines of the quasar Q 1101-264 [43] with the redshift $z=$ 1.839:

$$
\Delta \alpha / \alpha=(2.4 \pm 3.8) \times 10^{-6}, \quad z=1.839 .
$$

A unified sample of Fe II lines gave $\Delta \alpha / \alpha=(-0.4 \pm 1.5) \times 10^{-6}$ for two systems with $z=1.15$ and 1.839 . 
The use of radio and millimeter quasar absorption spectra gave an independent estimate (unfortunately, for comparatively small redshifts only)

$$
|\Delta \alpha / \alpha|<8.5 \times 10^{-6}, \quad z=0.25-0.68
$$

As noted by Barrow [44], the same quasar absorption spectra analysis leads to an upper bound on spatial variations of $\alpha$ like $|\Delta \alpha| / \alpha<3 \times 10^{-6}$ at 3 Gpc distance scale because of a wide distribution of the target absorption systems over the sky.

An alternative method is to study emission rather than absorption spectra. There are very few such estimates since this method, being sufficiently simple and direct, is less sensitive and is harder to apply to sources with large redshifts. Recent measurements of strong emission lines of O III in a sample of 165 quasars have led to the result [45]

$$
\Delta \alpha / \alpha=(1.2 \pm 0.7) \times 10^{-4}, \quad z=0.16-0.8 .
$$

Essential improvements in emission line measurements and analysis is anticipated in the near future [46], partly owing to the use of many-multiplet methods already employed in the absorption spectra analysis.

The estimate (17) as well as tentative estimates of the Cambridge group (England) [47] point at higher values of $\alpha$ in the past whereas the results related to absorption lines give preference to smaller $\alpha$ in the past. It appears tempting to conclude that this apparent discrepancy may be explained by some unidentified systematic errors. Arguments in favour of this viewpoint have been put forward by Bandeira and Corbelli [48], who found that at least the absorption line analysis could be subject to systematic effects, related to estimation of different sets of atomic transitions at different redshifts, and these hidden correlations thus could mimic a variable $\alpha$.

Another, "optimistic" viewpoint is, however, possible [47], that this is simply an indication of spatial variations of $\alpha$ since the emission and absorption methods are sensitive to the values of $\alpha$ in drastically different environments.

One can conclude that the present astrophysical data on $\alpha$ variation are rather contradictory and need further verification and improvement.

\subsection{Cosmological data}

The observed anisotropy of the cosmic microwave background (CMB) and the abundance of light elements formed in primordial nucleosynthesis (PNS) constrain the variations of $\alpha$ on the cosmological scale on the level $10^{-2}$. These constraints concern much greater redshifts $z$ than any others: $z \sim 1000$ for CMB and $z \sim 10^{10}$ for PNS.

Variations of $\alpha$ change the Thomson scattering cross-section and hence the transparency of the medium and finally the recombination time.

A recent analysis of the WMAP (Wilkinson Microwave Anisotropy Probe) data on CMB anisotropy has led to the result [49]

$$
\Delta \alpha / \alpha=(-1.5 \pm 3.5) \times 10^{-2},
$$

for $z \sim 10^{3}$. It is, however, well known that a similar effect on CMB anisotropy could be due to variations of the gravitational constant [50]. Besides, consistent constraints on $\alpha$ variations of the order of $1 \%$ could only be obtained from CMB analysis combined with independent 
data on the cosmological parameters characterizing the expansion of the Universe at times close to the recombination epoch.

Barrow [44] used the observed CMB isotropy at large angular scales to derive strong observational limits on any possible large-scale spatial variation in the values of $\alpha$ and other FPC (the electron to proton mass ratio and the Newtonian gravitational constant) assuming that their space-time evolution is driven by a scalar field. The constraints are strongly model-dependent. Thus, large-scale spatial fluctuations of $\alpha$ are bounded by $2 \times 10^{-9}$ in the BSBM theory (see below) and by $1.2 \times 10^{-8}$ in the varying speed of light theories. These derived bounds are significantly stronger than any others, obtainable by direct observations of astrophysical objects. It should be noted, however, that these bounds concern variations between mutually remote regions of space with approximately equal physical conditions and do not apply to the possible discrepancy between the results of emission and absorption spectra of quasars (see the end of the previous section): in the latter case, the emitters and absorbers are apparently characterized by drastically different gravitational fields, matter densities and temperatures.

The PNS theory predicts light element formation in the early Universe, and their resulting abundances depend on a delicate balance between the Universe expansion and the weak interaction strength which in turn determines the proton to neutron number ratio at the beginning of PNS. Ultimately, the predicted light element abundances depend on a number of fundamental constants, $\alpha$ being only one of them.

A recent study incorporating seven parameters [51] has yielded

$$
\Delta \alpha / \alpha=(6 \pm 4) \times 10^{-4}, \quad z \sim 10^{10} .
$$

A considerable improvement of the above estimates is expected with the appearance and analysis of new observational data from the WMAP and Planck Surveyor satellites.

According to Ref. [47], the CMB and PNS analysis on the whole favours slightly smaller values of $\alpha$ in the past compared to its modern value. The absence of variations also remains admissible on the level of $2 \sigma$.

The bounds (18) and (19) cannot be directly translated into estimates of the admissible current values of $\dot{\alpha}$ since, to do so, one has to assume a certain dependence $\alpha(t)$ which is not only different in different theories, but even in specific models of a given theory; see examples in Sec. 3.

\subsection{Comparing data of different origin}

The above discussion of possible FPC variability combined observational evidence from quasar spectra with Solar system data and laboratory constraints. Most of the studies implicitly assume that local and cosmological observations are directly comparable. This is, however, a strong assumption which may prove to be wrong. If a given FPC, say, $\alpha$, depends on a scalar field $\phi$, a slowly varying cosmological background of $\phi$ may substantially differ from its local values in a specific galaxy, stellar cluster or another gravitationally bound object. One can freely imagine, for instance, that the Oklo data only reflect the value of a stationary galactic scalar field whereas the quasar data feel cosmological effects.

Shaw and Barrow [52] have found that it is probably not the case, and local variations of the "constants" are able to track their global variations. A construction involving matched 
asymptotic expansions within a sufficiently wide scalar field model is analyzed assuming that the scalar (dilaton) field is only weakly coupled to gravity and has a negligible effect on the background space-time geometry; a local inhomogeneity was characterized by the Tolman-Bondi class of spherically symmetric metrics describing the evolution of a dust cloud in the presence of a cosmological constant. It was concluded that local virialization does not stabilize the value of the dilaton and protect it from any global cosmological variation. This indicates that local terrestrial and Solar-system experiments really measure the effects of varying "constants" of Nature occurring on cosmological scales to computable precision [52].

Somewhat different results have been obtained by Mota and Barrow [53,54]. They studied the space and time evolution of $\alpha$ using the BSBM varying- $\alpha$ theory (see Sec. 3.1) and a spherical collapse model for cosmological structure formation, considered as a perturbation to a homogeneous and isotropic cosmological background. Different assumptions were used on the dark energy equation of state and on the coupling of $\alpha$ to the matter fields. It was found that, independently of the model of structure formation one considers, there is always a difference between the values of alpha in a virialized overdensity and in the background. In some models, especially at low redshifts, the difference depends on the time when virialization has occurred and the equation of state of the dark energy. At low redshifts, when the dark energy starts to dominate the cosmological expansion, the difference between alpha in a cluster and in the background grows.

According to these studies, it may happen that $\alpha$ and $\dot{\alpha}$ vary from galaxy to galaxy even at the same redshift, but the evolution of $\alpha$ is the same in different sub-galactic stellar systems belonging to a single galaxy, or even in galaxies belonging to the same galaxy cluster [55]. It is, however, clear that the problem should be studied more thoroughly in a wider range of models.

More generally, one can conclude that the whole set of modern experimental and observational data leads to very stringent limits of putative $\alpha$ variations but leaves open the question of their real existence. Even more uncertainty follows from the possible difference between local and global $\alpha$ variations and its model dependence.

\section{Theoretical models that predict varying $\alpha$}

Despite the weak experimental status of varying $\alpha$, there have been a great number of theoretical studies in the recent years, treating $\alpha$ as a function of certain physical fields which, in general, change from point to point in four-dimensional space-time.

It should be noted that the researchers themselves stress a tentative nature of such constructions. Thus, according to [47], "any model compatible with all empirical data at a given time, is certainly wrong since at any time some of the current data are erroneous". It is therefore useless to build artificial models with a large number of free parameters: it is highly probable that such a model will be incompatible with the newest data as soon as its description is published.

In studies dealing with multidimensional theories, such as Kaluza-Klein theories and different versions of string theory (regarded at present as the most promising unification theories), the dimensionless constants of four-dimensional physics are shown to be dynamical quantities $[56,57]$. However, the specific form of such dynamics not only depends on the 
version of the Kaluza-Klein or string theory, but also on the compactification scheme and the form of dilatonic coupling adopted within the chosen version of the theory.

Accordingly, at the present stage, comparatively simple and natural phenomenological models are preferable, which, on the one hand, should conform to the idea of unity of all physical interactions and, on the other, should not only be able to predict the variability of $\alpha$ but also suggest solutions to other problems of modern cosmology, such as the cosmological constant problem and the dark matter and dark energy problems. We will briefly outline two such models and mention some other approaches.

\subsection{Bekenstein-Sandvik-Barrow-Magueijo (BSBM) theories}

This class of theories $[58,59]$ assumes constant values of the speed of light and Planck's constant; accordingly, a variability of $\alpha$ is achieved due to variability of the electron charge $e$ or the permittivity of vacuum. Thus instead of $e=$ const ane assumes $e=e_{0} \varepsilon(x)$, where $e_{0}=$ const while $\varepsilon$ is a dimensionless scalar field depending on the space-time coordinates. Such theories preserve the usual properties of local gauge and Lorentz invariance and causality. In these theories, the conventional expression for the electromagnetic field tensor in terms of the 4 -potential $A_{\mu}$ is replaced by

$$
F_{\mu \nu}=(1 / \varepsilon)\left[\left(\varepsilon A_{\nu}\right)_{, \mu}-\left(\varepsilon A_{\mu}\right)_{, \nu}\right]
$$

which takes its usual form if $\varepsilon=$ const. The electromagnetic field action has its usual form while the scalar field dynamics is described by the usual kinetic term in the Lagrangian (written in the form $\left.\varepsilon_{, \mu} \varepsilon^{\mu} / \varepsilon^{2}\right)$. The theories of this class differ from one another in assumptions on how the $\varepsilon$ field is coupled to the rest of matter.

The cosmological models obtained in these theories predict time variations of $\alpha$ (e.g., $\sim \ln t$ in the matter-dominated phase in a spatially flat cosmology) and spatial variations of $\alpha$ whose nature and evolution depends on the dark energy equation of state and the coupling of $\alpha$ to the matter fields $[53,54]$. Inclusion of the effects of inhomogeneity, so that the dependence $\alpha(t)$ differs in the cosmological background and in virialized overdensities and may naturally lead to no observable local time variations of $\alpha$ on Earth and in our Galaxy even though such variations can be significant on quasar scales.

\subsection{Fujii's scalar-tensor theory}

The theory suggested by Fujii [60] contains, in addition to the metric tensor, two scalar fields. One of them $(\sigma)$ is a dilatonic Brans-Dicke type field with the potential $V(\sigma)=\Lambda \mathrm{e}^{-4 \zeta \sigma}$ (in the Einstein conformal frame, or picture), where $\Lambda$ is a constant playing the role of a cosmological constant in the Jordan picture; $\zeta$ is the so-called non-minimal coupling constant characterizing a coupling between the $\sigma$ field and the metric in the Jordan picture. Another scalar field $\phi$ has a conventional kinetic term and interacts with $\sigma$ in such a way that their common potential in Einstein's picture has the form

$$
V(\phi, \sigma)=\mathrm{e}^{-4 \zeta \sigma}\left[\Lambda+\frac{1}{2} m^{2} \phi^{2}(1+\gamma \sin (k \sigma))\right],
$$

where $m, \gamma, k$ are constants (approximately of order unity in the Planck system of units).

Considering Jordan's picture as a physical one, the author studies the modification of standard quantum electrodynamics in this theory caused by the scalar fields and concludes 
that the effective electric charges are $\sigma$-dependent. The time dependence of $\sigma$ itself is affected by the $\phi$ field which leads to decaying oscillations of $\sigma$ in the modern epoch.

A cosmological model built in the framework of this theory predicts comparatively rapid changes of $\alpha$ in the epoch corresponding to $z>1$ (but close enough to 1) and much slower changes in the modern epoch. This allows one to reconcile the strong bounds related to the Oklo phenomenon and the conclusions $[12,40]$ on rather a rapid growth of $\alpha$ in the previous epoch. The field $\sigma$ is also able to play the role of the so-called quintessence explaining the Universe acceleration observed in modern cosmology.

Other predictions of Fujii's theory are the existence of a non-Newtonian short-range component of gravity and a weak equivalence principle violation. The orders of magnitude of both effects are compatible with modern experimental constraints.

\subsection{Other models}

We have briefly described two sufficiently well-known and elaborated phenomenological theories. Let us also mention some alternative approaches.

Anchordoqui and Goldberg [61] discussed the so-called quintessence, i.e., a minimally coupled scalar field playing the role of an "accelerator" of the Universe expansion, as a possible generator of $\alpha$ variability. They considered scalar field potentials combining a number of exponential functions with different coefficients. Like Fujii's theory, this model makes it possible to reconcile the tough constraints obtained from the Oklo reactor with the results of Webb et al. obtained from quasar absorption spectra: they become compatible due to slowing down of the scalar field evolution in approaching the present epoch.

Damour and Polyakov [62] suggest a more general approach: among the models of the Universe containing ordinary matter and a real scalar field, one singles out a class of lowenergy models possessing invariance under diffeomorphisms and gauge transformations. The resulting theories have a Born-Infeld type Lagrangian structure. In such theories, the masses and $g$-factors of fermions, as well as their electromagnetic coupling constants, are scalar field dependent, whereas their electric charges and gravitational coupling may remain constant. One then analyzes the effect of all variable factors on the observed atomic spectra. The latter, as follows from Ref. [62], bear information on the possible variability of $\alpha$ as well as the $g$-factors and particle mass ratios, and, moreover, all predictions concerning the atomic spectra turn out to be conformal gauge independent.

Bertolami et al. [63], among others, stress the effective Lorentz and CPT symmetry violation that accompanies variable fundamental constants in any theory where these constants are scalar field dependent since variable scalar fields inevitably create preferred space-time directions. As en example of such a theory, they analyze $\mathrm{N}=4$ supergravity in four dimensions, containing two scalar fields, and show that some of its solutions lead to $\alpha(t)$ dependence compatible with the astrophysical data, though at the expense of some fine tuning. In another paper [64], Bento, Bertolami and Santos considered a cosmological model with a two-field quintessence, i.e., a set of two scalar fields $\phi$ and $\psi$, minimally coupled to the Einstein gravity and possessing the potential

$$
V(\phi, \psi)=\mathrm{e}^{-\lambda \psi} P(\phi, \psi)
$$

where $P(\phi, \psi)$ is a third-order polynomial depending on both fields, while the electromagnetic 
field Lagrangian had the form

$$
L_{\mathrm{e}-\mathrm{m}}=\frac{1}{4} B(\phi, \psi) F^{\mu \nu} F_{\mu \nu}
$$

$B(\phi, \psi)$ being a linear function of both scalars. It was found that some solutions for this system describe a cosmology with a transient late period of accelerated expansion, making it possible to fit the data arising from quasar absorption spectra and comply with the bounds on the variation of $\alpha$ from the Oklo reactor, meteorite analysis, atomic clock measurements, cosmic microwave background radiation, and big bang nucleosynthesis.

Kirillov (see [65] and references therein) suggested a modified field theory which is aimed at explaining all effects ascribed to dark matter and dark energy without explicitly introducing them. A phenomenological manifestation of this theory may be expressed as a modification of the law of gravity, such that, for a point source of mass $M_{0}$, the gravitational potential reads

$$
\phi=-\frac{G M_{0}}{r}[1+f(r)]
$$

where the function $f(r)$ may be chosen in the form

$$
f(r)= \begin{cases}\left(r / r_{0}\right) \ln \left(r_{\max } \mathrm{e} / r\right), & r \leq r_{\max }, \\ r_{\max } / r_{0}, & r>r_{\max }\end{cases}
$$

the parameter $r_{0}$, having the order of $1-5 \mathrm{kpc}$, is different for different galaxies while $r_{\max }$ only depends on cosmological time and is now of the order of $100 \mathrm{Mpc}$. At $r>r_{\max }$, Newton's law is restored but with an effective mass $M_{\max }=M_{0}\left(1+r_{\max } / r_{0}\right)$. It is shown that such a modification is able to account for all dark matter effects observed and, in particular, to explain the whole variety of "dark matter halos" in astrophysical systems. It is also shown that, in the modified theory, the galaxy formation process leads to an effective equaton of state of the fictituous "dark matter" with $w<-1 / 3$ ( $w$ being the ratio of pressure to energy density) and therefore it can play the role of dark energy. The standard picture of the early Universe is preserved, but the theory predicts variation of all interaction constants (including $G$ and $\alpha$ ) with the same time dependence [66].

\section{Concluding remarks. Physical and metrological significance of FPC variations}

General theoretical considerations related to the necessary unification of all interactions and the requirement of unity of the physical science lead, probably inevitably, to the idea of a dynamical nature of all known FPCs or at least a greater part of them. Thus more surprising is that so much effort is needed to discover their variability than the belief that they are variable. Meanwhile, the experimental confirmations of these ideas are at present very poor and unreliable. This certainly concerns all FPCs, not only $\alpha$.

An important and natural feature of all models is the interrelation of the predicted variations of different FPCs. Therefore, in both planning and interpretation of future experiments, such interrelations should be taken into account. Thus, in the context of string theories, it 
turns out [62] that the quantum chromodynamics constant $\Lambda_{\mathrm{QCD}}$ and the weak interaction constant $v$ are "even more variable" than $\alpha[15]$ :

$$
\Delta \Lambda_{\mathrm{QCD}} / \Lambda_{\mathrm{QCD}} \sim 30 \Delta \alpha / \alpha, \quad \Delta v / v \sim 80 \Delta \alpha / \alpha
$$

According to [67], any theoretical model, predicting cosmological variations of $\alpha$ over $10^{-6}$ due to changes of some long-range scalar field, also predicts violations of the weak equivalence principle (WEP) over $10^{-13}$. To discover such variations, it is only necessary to gain one order of magnitude as compared to the modern experimental constraints. This endows special significance to the space experiments for testing the WEP planned for the nearest years: MICROSCOPE (planned sensitivity $10^{-15}$ ), STEP (up to $10^{-18}$ [68]) and others.

Thus new space experiments and observations in the area of gravitation, cosmology and astrophysics (improved satellite observations of the microwave background, analysis of supernovae and quasar radiation, space experiments for testing the WEP and constancy of the gravitational constant $G$, laboratary tests of FPC stability etc.) are expected to bring new results of major significance for the whole physical picture of the world. The discovery of a dynamical nature of a number of FPCs, as well as revision of the level of fundamentality for many of them, will inevitably lead to the corresponding revision in the foundations of theoretical metrology. Following the change in the general theoretical paradigm, the set of FPCs will also be revised: the parameters, whose dynamical nature will be proved, will lose their fundamental status, giving way to the basic constants of an underlying unified theory.

If $\alpha$ does vary, a question of great interest for both physics and metrology is: which of the quantities in the definition (1) of $\alpha$, namely, $c, \hbar, e$ or $\varepsilon_{0}$, are really varying. Different viewpoints may be found in the literature.

The theories mentioned in Sec. 3 modify the Maxwell equations and actually introduce a scalar field dependence of the effective electronic charge $e$. On the other hand, Hehl and Obukhov [69] note that, since $e$ and $h$, as the units of charge and action, are invariants (4D scalars), to reconcile a variable $\alpha$ with the invariable (premetric) form of the Maxwell equations one may choose the speed of light $c$ to vary. For variable $c$ theories see, e.g., Ref. [70], the brief review [71] and references therein. Though, as noted by Alfonso-Faus [72], such first principles as local Lorentz invariance and local position invariance disfavour variations of $c$ as compared to changes of $e$ as a reason for varying $\alpha$.

Hehl and Obukhov [69] mention one more possibility: they represent $\alpha$ as

$$
\alpha=e^{2} \Omega_{0} /(4 \pi \hbar)=\Omega_{0} /\left(2 R_{K}\right)
$$

where $\Omega_{0}$ is the impedance of free space and $R_{K}$ is the von Klitzing constant (quantum Hall resistance). In these expressions, the speed of light $c$ has disappeared. If, again, $e$ and $\hbar$ are regarded real constants, the putative $\alpha$ variability should be attributed to the vacuum impedance $\Omega_{0}$ that becomes a dynamic (dilaton) field.

Tobar [27] represented $\alpha$ in terms of the ratio of the quanta of electric $\left(\Phi_{e}\right)$ and magnetic $\left(\Phi_{m}\right)$ fluxes of force of the electron:

$$
\alpha=\frac{1}{4 \sqrt{2}}\left(\frac{\Phi_{e}}{\Phi_{m}}\right)^{1 / 2} .
$$


The quantity $\alpha$, being dimensionless, is independent of the system of units used. Eq. (28) makes this independence manifest, which is an advantage as compared with the conventional expressions (1). The representation (28) made it possible [27] to interpret the range variation of $\alpha$ at the electroweak energy scale (see the end of Sec. 2.1) as appearing due to equal components of electric screening (polarization of vacuum) and magnetic anti-screening (magnetization of vacuum), which cause the perceived quanta of electric charge to increase at small distances, while the magnetic flux quanta decrease. Thus there emerge the concepts of "bare magnetic flux quanta" and "bare electric charge". With regard to a putative drift at astrophysical or cosmological scales, Tobar [27] also interpreted it as a differential drift of the electric and magnetic fluxes, but concluded that it is impossible to determine which fundamental constant is varying if $\alpha$ varies.

In our view, the constants $c$, related to the space-time structure, and $\hbar$, related to the quantum properties of all kinds of matter, are more universal than $e$ that characterizes the electromagnetic interaction only and are therefore more likely to be true constants. It is quite natural that unification theories modify the Maxwell equations by introducing slowly varying scalars which make the effective electronic charge vary. This view is also consistent with HehlObukhov's and Tobar's interpretations. On the other hand, the constant $\varepsilon_{0}$, which is present in the SI definition of the fine structure constant, only connects the electric SI units with the mechanical ones and does not have a fundamental meaning. (See also a recent discussion of SI and other systems of units in Refs. [73,74].)

Thus a variable $\alpha$ raises a number of questions in fundamental metrology, requiring further experimental and theoretical studies.

As to the needs of practical metrology, the existing constraints on possible variations of $\alpha$ in the modern epoch, no more than a few units of the 17th significant digit per year [see Eq. (9)], compared to the achieved measurement accuracy of $10^{9}$, give no ground to expect any appreciable change in this constant in the foreseeable future. Thus there is no evident reason for taking into account the conjectural dynamical nature of $\alpha$ in the values of references of any physical quantities, their construction and analysis. However, as follows from our discussion, there are at least two exceptions: (i) observational and theoretical cosmology and astrophysics of remote quasars and galaxies, and (ii) high energy physics related to unification of interactions.

A problem of importance for both theoretical and practical metrology is the connection between local and cosmological variations of the FPCs, $\alpha$ in particular. Such a connection should be known for comparison between, e.g., terrestrial and quasar measurements of $\alpha$ and $\dot{\alpha}$ and their proper interpretation. On the other hand, since this connection appears to be model-dependent [52-54], spatial variations of FPCs deserve special attention due to their potential use for model selection in physics and cosmology.

\section{Acknowledgment}

The authors are grateful to L.K. Isaev, V.N. Melnikov and M.I. Kalinin for valuable discussions. K.B. thanks David Mota for helpful correspondence. 


\section{References}

[1] K.P. Staniukovich, "Gravitational Field and Elementary Particles", Moscow, Nauka, 1965.

[2] K.P. Staniukovich and V.N. Melnikov, "Hydrodynamics, Fields and Constants in the Theory of Gravitation", Energoatomizdat, Moscow, 1983 (in Russian);

V.N. Melnikov, "Fields and Constants in the Theory of Gravitation", CBPF-MO-02/02, CBPF, Rio de Janeiro, 2002, 134pp.

[3] V. De Sabbata, V.N. Melnikov and P.I. Pronin. "Theoretical Approach to Treatment of Nonnewtonian Interactions", Progr. Theor. Phys. 88, 623 (1992).

[4] K.A. Bronnikov, V.D. Ivashchuk and V.N. Melnikov, "Time variation of the gravitational constant in multidimensional cosmology", Nuovo Cim., B102, 209-216 (1988).

[5] K.A. Bronnikov, V.N. Melnikov and Mario Novello, "Possible time variations of $G$ in scalartensor theories of gravity" Grav. 85 Cosmol. 8, Suppl. II (2002) (Proc. ICGA-5, Moscow, 1-7 October 2001), pp. 18-21.

[6] V.N. Melnikov, in: "Gravitational Measurements, Fundamental Metrology and Constants", eds. V. de Sabbata and V.N. Melnikov, Kluwer Academic Publ., Dordtrecht, 1988, p. 283.

[7] V.N. Melnikov, Int. J. Theor. Phys. 33, 1569 (1994).

[8] V.N. Melnikov. "Gravity as a key problem of the millennium". Proc. 2000 NASA/JPL Conference on Fundamental Physics in Microgravity, Solvang, CA, USA. NASA Document D-21522, 2001, p. 4.1-4.17.

[9] K.A. Bronnikov and V.N. Melnikov, "Conformal frames and D-dimensional gravity", in: Proceedings of the 18th Course of the School on Cosmology and Gravitation: The Gravitational Constant. Generalized Gravitational Theories and Experiments (30 April-10 May 2003, Erice), Ed. G.T. Gillies, V.N. Melnikov and V. de Sabbata, Kluwer, Dordrecht/Boston/London, 2004, pp. 39-64; gr-qc/0310112.

[10] S.A. Kononogov and V.N. Melnikov, Izm. Tekhnika 6, 1 (2005); Meas. Techniques 48, 6, 521 (2005).

[11] N.I. Kolosnitsyn, S.B. Pushkin and V.M. Purto, "Experimental test of a possible variation of fundamental physical quantities", in: "Problems of Gravitation Theory and Particle Theory", 7th issue, ed. K.P. Staniukovich, Atomizdat, Moscow, 1976, p. 208-211 (in Russian).

[12] J. Webb et al., Phys. Rev. Lett. 82, 884 (1999).

[13] J.Ph. Uzan, Rev. Mod. Phys. 75, 403 (2003).

[14] S.G. Karshenboim, Gen. Rel. Grav. 38, 159-182 (2006); physics/0311080

[15] J.-Ph. Uzan, astro-ph/0409424. Variation of the constants in the late and early universe Invited talk at the conference "The Quest for Cosmological Scalar Fields", Porto, 8-10 July 2004.

[16] J.P. Turneaure and S.R. Stein, in: "Atomic Masses and Fundamental Constants 5", ed. J.H. Sanders and A.H. Wapstra, Plenum, NY, 1976, p. 636.

[17] B.W. Petley, "The Fundamental Physical Constants and the Frontier of Measurement", Adam Hilger, Bristol-Boston, 1985.

[18] H. Marion et al., Phys. Rev. Lett. 90, 150801 (2003).

[19] S. Bize et al., Phys. Rev. Lett. 90, 150802 (2003).

[20] M. Fischer et al., Phys. Rev. Lett. 92, 230802 (2004). 
[21] E. Peik et al., Phys. Rev. Lett. 93, 170801 (2004), physics/0402132; Laser Physics 15, 1028 (2005), physics/0504101.

[22] O.Yu. Andreev, L.N. Labzowsky, G. Plunien and G. Soff, "Testing the time dependence of the fundamental constants in the spectra of multicharged ions", physics/0505081.

[23] S.G. Karshenboim et al., "A new option for a search for alpha variation: narrow transitions with enhanced sensitivity", J. Phys. B. At. Mol. Opt. Phys., 39, 1937-1944 (2006), physics/0511180.

[24] S. Bize et al., "Cold atom clocks and applications", physics/0502117.

[25] C. Salomon et al., C.R. Acad. Sci. Paris, T2 Série 4, 1313 (2001).

[26] I. Levine et al., Phys. Rev. Lett. 78, 424 (1997).

[27] M.T. Tobar, Metrologia 42, 129 (2005).

[28] A.I. Shlyakhter, Nature (London) 264, 340 (1976).

[29] T. Damour and F. Dyson, Nucl. Phys. B 480, 37 (1996).

[30] Y. Fujii et al., Nucl. Phys. B 573, 377 (2000).

[31] S.K. Lamoreaux and J.R. Torgerson, Phys. Rev. D 69, 121701 (2004).

[32] V.V. Flambaum and E.V. Shuryak, Phys. Rev. D 67, 083507 (2002).

[33] K. Olive et al., Phys. Rev. D 66, 045022 (2002).

[34] S.K. Lamoreaux, Phys. Rev. D 69, 121701 (2004). nucl-th/0309048.

[35] Yu.V. Petrov et al., "Natural nuclear reactor Oklo and variation of fundamental constants. Part 1: computation of neutronic of fresh core", hep-ph/0506186.

[36] P.J.E. Peebles and R. Dicke, Phys. Rev. 128, 2006 (1962).

[37] K. Olive et al., Phys. Rev. D 69, 027701 (2000).

[38] M.T. Murphy et al., Month. Not. R. Astron. Soc. 345, 609 (2003).

[39] H. Chand et al., Probing the time-variation of the fine-structure constant: Results based on $\mathrm{Si}$ IV doublets from a UVES sample. astro-ph/0408200.

[40] J. Webb et al., Phys. Rev. Lett. 87, 091301 (2001).

[41] M.T. Murphy et al., Month. Not. R. Astron. Soc. 327, 1237 (2003).

[42] R. Quast et al., Astron. Astrophys. 415, L7 (2004).

[43] S.A. Levshakov et al., "VLT/UVES constraints on the cosmological variability of the finestructure constant", astro-ph/0408188.

[44] J.D. Barrow, Phys. Rev. D 71, 083520 (2005); astro-ph/0503434.

[45] J. Bahcall et al., Astroph. J. 600, 520 (2004).

[46] D. Grupe, A.K. Pradhan and S. Frank, "Studying the variation of the fine structure constant using emission line multiplets", astro-ph/0504027.

[47] C.J.A.P. Martins, "New constraints on varying alpha", astro-ph/0405630.

[48] R. Bandeira and E. Corbelli, Astron. Astrophys. 434, 543 (2005); astro-ph/ 0504340

[49] C.J.A.P. Martins et al., Phys. Lett. B 585, 29 (2004).

[50] A. Riazuelo and J.-P. Uzan, Phys. Rev. D 66, 023525 (2002).

[51] C.M. Müller, G. Schäfer and C. Wetterich, Phys. Rev. D 70, 083504 (2004), astro-ph/0405373. 
[52] D.J. Shaw and J.D. Barrow, "Local experiments see cosmologically varying constants", Phys. Lett. B 639, 596-599 (2006); gr-qc/0512117.

[53] D.F. Mota and J.D. Barrow, "Local and global variations of the fine structure constant", Mon. Not. Roy. Astron. Soc. 349, 291 (2004).

[54] D.F. Mota and J.D. Barrow, "Varying alpha in a more realistic universe", Phys. Lett. B 581, 141 (2004).

[55] D.F. Mota, 2006, private communication.

[56] T.R. Taylor and G. Veneziano, Phys. Lett. B 213, 450 (1988); E. Witten, Phys. Lett. B 149, 351 (1984).

[57] J. Polchinski, "Superstring theory", Cambridge University Press, 1997.

[58] J.D. Bekenstein, Phys. Rev. D 25, 1527 (1982).

[59] J.D. Barrow, J. Magueijo and H.D. Sandvik, Phys. Rev. D 66, 043515 (2002).

[60] Y. Fujii, Int. J. Mod. Phys. D 11, 1137 (2002); Grav. Cosmol. 6, 107 (2000); Phys. Rev. D 62, 044011 (2000).

[61] L. Anchordoqui and H. Goldberg, Phys. Rev. D 68, 083513 (2003), hep-ph/0306084.

[62] T. Damour and A.M. Polyakov, Nucl. Phys. B 423, 532 (1994).

[63] O. Bertolami, R. Lehnert, R. Potting and A. Ribeiro, Phys. Rev. D 69, 083513 (2004).

[64] M.C. Bento, O. Bertolami and N.M.C. Santos, Phys. Rev. D 70, 107304 (2004).

[65] A.A. Kirillov, Phys. Lett. B 632, 453-462 (2006), astro-ph/0505131.

[66] A.A. Kirillov, in: J. Val Blain (Ed.), Trends in Dark Matter Research, Nova Science Publishers, New York, 2005, pp. 1-38; astro-ph/0405623.

[67] T. Damour, Astrophys. Space Sci. 283, 445-456 (2003); gr-qc/0210059.

[68] P.W. Worden, in: Proc. 7th M. Grossmann Meeting on General Relativity, Stanford, 1994 (World Scientific, Singapore, 1996), p. 1569.

[69] F.W. Hehl and Yu.N. Obukhov, "Dimensions and units in electrodynamics", physics/0407022.

[70] A. Peres, Int. J. Mod. Phys. A 12, 1751 (2004); gr-qc/0210066.

[71] V.D. Ivashchuk and S.A. Kononogov, "On variation of the speed of light in modern gravitational models", Grav. Cosmol. 11, 259 (2005).

[72] A. Alfonso-Faus, "Implications of a time-varying fine structure constant", gr-qc/0212020.

[73] C.J. Borde, Comptes Rendus Physique 5, 813 (2004).

[74] S.A. Kononogov, "Metrology and fundamental physical constants", Meas. Techniques 49, 2, 97-102 (2006). 